\title{
U2OS cells lacking Chk1 undergo aberrant mitosis and fail to activate the spindle checkpoint
}

\author{
Laura Carrassa $^{a, *}$, Yolanda Sanchez ${ }^{b}$, Eugenio Erba ${ }^{a}$, Giovanna Damia ${ }^{a}$ \\ a Department of Oncology, Istituto di Ricerche Farmacologiche "Mario Negri", Milan, Italy \\ ${ }^{b}$ Department of Pharmacology and Toxicology, Dartmouth Medical School, Hanover, NH, USA
}

Received: November 16, 2007; Accepted: April 21, 2008

\begin{abstract}
Chk1 is a conserved protein kinase originally identified in fission yeast, required to delay entry of cells with damaged or unreplicated DNA into mitosis. The requirement of Chk1 for both S and G2/M checkpoints has been elucidated while only few studies have connected Chk1 to the mitotic spindle checkpoint. We used a small interference RNA strategy to investigate the role of Chk1 in unstressed conditions. Chk1 depletion in U20S human osteosarcoma cells inhibited cell proliferation and raised the percentage of cells with a 4N DNA content, which correlated with accumulation of giant polynucleated cells morphologically distinct from apoptotic cells, while no increased number of cells in G2 or mitosis could be detected. Down-regulation of Chk1 also caused accumulation of cells in the last step of cytokinesis, and of tetraploid cells in G1 phase, which coincided with activation of p53 and increased levels of p21. In addition, Chk1depleted U20S cells failed to arrest in mitosis after spindle disruption by nocodazole and showed decreased protein levels of Mad2 and BubR1. These studies show that U20S cells lacking Chk1 undergo abnormal mitosis and fail to activate the spindle checkpoint, suggesting a role of Chk1 in this checkpoint.
\end{abstract}

\section{Keywords: Chk1 $\bullet$ cell cycle $\bullet$ mitosis $\bullet$ spindle checkpoint $\bullet$ tetraploidy $\bullet$ siRNA}

\section{Introduction}

Chk1 is a conserved protein kinase. It was originally identified in fission yeast [1] and its role was then largely confirmed in vertebrate cells as a protein kinase required to delay entry of cells with damaged or unreplicated DNA into mitosis [2, 3]. The involvement of Chk1 protein kinase in transducing DNA damage signals and its requirement for both $S$ phase and G2/M checkpoint has been elucidated but few experimental evidence has recently connected Chk1 to the mitotic spindle checkpoint. This checkpoint ensures proper chromosome segregation by delaying anaphase until chromosomes are aligned on the spindle thus preventing chromosomal instability, aneuploidy and cancer predisposition [4].

It has been recently proposed that Chk1 is needed in metaphase for proper chromosome alignment on the equatorial planes, as shown by a pronounced block in mitosis of Chk1depleted HeLa cells [5]. Chk1-deficient cells also fail to sustain

\footnotetext{
*Correspondence to: Laura CARRASSA, Laboratory of Molecular Pharmacology, Department of Oncology, Istituto di Ricerche Farmacologiche

"Mario Negri", via La Masa 19, 20156, Milan, Italy.

Tel.: +39-02-39014237

Fax: $+39-02-3546277$

E-mail: carrassa@marionegri.it
}

mitotic arrest after treatment with taxol, which stabilizes microtubules, but do arrest in response to nocodazole (Noc), suggesting that Chk1 helps to sustain anaphase delay when chromosome biorientation is disrupted [6].

Although the role of Chk1 in DNA damage response has been largely clarified, its role during normal cell cycle progression remains controversial. Chk1 -/- mice die at E6.5 days [2] indicating its fundamental role in early embryonic development. Corroborating this, a conditional KO of Chk1 induced apoptosis in embryonic mice stem cells [7] and led to premature mitosis before completion of DNA replication and cell death by mitotic catastrophe [8]. However, evidence on the role of Chk1 in somatic cancer and in normal cell lines and tissues is controversial. The only available vertebrate Chk1 KO somatic cancer cellular system, the DT40 avian lymphoma cell line Chk1-/-, is viable despite multiple checkpoint and survival defects [9]. These data are in agreement with what has been reported in various human cancer cell lines where Chk1 depletion by siRNA did not alter the cell cycle profile or induce apoptosis in the absence of DNA damage $[10,11]$. In contrast, Chk1 is essential for growth of mammary proliferating epithelial cells and a strong phenotype was observed in heterozygosity with general mis-coordination of the cell cycle due to inappropriate S phase entry, accumulation of DNA damage during replication and failure to restrain mitotic entry 
[12]. Similar results were recently obtained in U20S cells in which Chk1 was depleted either by specific inhibitors or by siRNA, where aberrant increased intiation of DNA replication was coupled with generation of DNA strand breaks and H2AX phosphorylation, although no abnormal cell morphology or investigation of cell survival at longer time-points after Chk1 inhibition were described [13].

We report here that U20S cells with Chk1 depleted by siRNA show an accumulation of polynucleated cells morphologically distinct from apoptotic cells. In addition, there is accumulation of cells with a 4N DNA content not associated with an increased number of mitotic cells and a failure to arrest in mitosis after treatment with Noc. These data led to the hypothesis that Chk1 function could be required for activation of the mitotic spindle checkpoint pathway. The results reported here open up an additional level of complexity on the role of Chk1 during normal growth, suggesting that depletion of Chk1 in somatic cancer and normal cell lines may have different consequences depending on the genetic background of the cell lines.

\section{Materials and methods}

\section{Cell culture and transfection}

Human U20S osteosarcoma cells were grown in Dulbecco's modified Eagle medium supplemented with $10 \%$ foetal bovine serum. Cells were cultured at $37^{\circ} \mathrm{C}$ in $5 \% \mathrm{CO}_{2}$. U2OS cells were treated with $75 \mathrm{ng} / \mathrm{ml}$ of Noc (Sigma, St Louis, MO, USA) for $20 \mathrm{hrs,} \mathrm{then} \mathrm{washed} \mathrm{twice} \mathrm{in} \mathrm{phosphate}$ buffered saline (PBS) and either starved or recovered in fresh medium and stored in the incubator for another $7 \mathrm{hrs}$. Staurosporine treatment was performed for $18 \mathrm{hrs}$ at a concentration of $50 \mathrm{nM}$.

\section{Small interfering RNA}

The Chk1 siRNA duplex target sequence used to deplete Chk1 expression in U2OS cells is $5^{\prime}$ AAGGGATAACCTCAAAATCTC $3^{\prime}$. A mismatch in $5^{\prime}$ of the antisense sequence of the double strand RNA was introduced to make the down-regulation more efficient, as already described by others [14]. The siRNA scramble sequence of Chk1 is $5^{\prime}$ AAACTATAAGGGTCAACACCT $3^{\prime}$. The siRNA duplexes were synthesized and purchased from Proligo-Sigma Genosys (Evry Cedex, France). To transfect siRNA duplexes, Lipofectamine 2000 Reagent (Invitrogen, Carlsbad, CA, USA) was used, following the manufacturer's instructions.

\section{Western blot analysis}

Cells were lysed in ice-cold whole cell extract buffer containing $50 \mathrm{mM}$ Tris $\mathrm{HCl} \mathrm{pH} 7.4,250 \mathrm{mM} \mathrm{NaCl}, 0.1 \%$ Nonidet NP40, $5 \mathrm{mM}$ EDTA and NaF $50 \mathrm{mM}$ with a protease inhibitor cocktail (Sigma). Lysates were cleared by centrifuging at 13,000 rpm for $5 \mathrm{~min}$. Around 30 micrograms of total protein extracts were loaded on $10 \%$ SDS-PAGE ( $6 \%$ of SDS-PAGE to detect BubR1), then transferred to a nitrocellulose membrane.

The polyclonal-sheep antibody against BubR1 was kindly provided by Doctor Stephen Taylor (Manchester University, UK). The mouse monoclonal
anti-Ran (clone 20) is from BD Transduction Laboratories (Erembodegem, Belgium). The rabbit phospho-p53 (ser15) antibody is from Cell Signaling Technology (Danvers, MA, USA). Other primary antibodies were purchased from Santa Cruz Biotechnology (Santa Cruz, CA, USA): anti-Chk1 (G4), p53 (D0-1), cyclin E (HE12), p21 (C-19), Mad2 (FL-205), geminin (FL-209) and actin (C-11). Densitometric analysis was done with Image J.

\section{Immunofluorescence analysis}

To stain with phosphorylated $\gamma \mathrm{H} 2 \mathrm{AX}$, U2OS cells were fixed at indicated time-points with methanol free formaldehyde in PBS $1 \%$ for 30 min. on ice, then in $70 \%$ ethanol. Before staining, cells were washed in PBS and treated with a permeabilization solution (0.5\% saponin, $10 \mathrm{mM}$ HEPES, $2.5 \mathrm{mM}$ $\mathrm{CaCl}_{2}$ and $1.4 \mathrm{M} \mathrm{NaCl}$ ) for $30 \mathrm{~min}$. at room temperature. Cells were then stained with monoclonal anti-phospho H2AX ( $\gamma \mathrm{H} 2 \mathrm{AX}$ ser-139, UPSTATE Upstate Technology, Lake Piacid, NY, USA). Antimouse IgG Alexa Fluor 594 (Molecular Probes Eugene, OR, USA) was used as secondary antibody for 30 min. at room temperature, 1:500 dilution, after three washes in PBS. To stain with phosphorylated serine 10 Histone 3 , LaminB or Aurora B, siRNA scramble and siRNA Chk1 transfected cells were fixed 72 hrs after transfection with $4 \%$ paraformaldehyde (in PBS solution) for 20 min. at room temperature, washed twice in PBS and stored at $4{ }^{\circ} \mathrm{C}$. Just before staining cells were permeabilized for $5 \mathrm{~min}$. with Triton X-100 (0.5\% in PBS) then blocked in Blocking Buffer (2\% BSA, 0.2\% triton X-100 in PBS) for $1 \mathrm{hr}$. Monoclonal anti-histone $\mathrm{H} 3$ phosphorylated at serine 10 (6G3, Cell Signaling Technology) diluted 1:150 was used to stain condensed chromosomes. Polyclonal antilamin B (goat, Santa Cruz Biotechnology) diluted 1:500 was used to stain the nuclear membrane envelope. The mouse monoclonal Anti-Aurora-B (AIM1) is from BD Transduction Laboratories.

After each of the above stainings, cells were incubated with DAPI (final concentration $30 \mathrm{ng} / \mathrm{ml}$ in PBS) for 1 min. to stain nuclei, then mounted with $7 \mu \mathrm{l}$ of Vectashield solution (Vector Laboratories, Burlingame, CA, USA). To stain with sulforodamine/DAPI solution, after 72 hrs siRNA scramble or siRNA Chk1 transfected cells were incubated for $30 \mathrm{~min}$. in the dark in a solution of DAPI and sulforodamine dissolved in $\mathrm{Na}-\mathrm{Tris} \mathrm{HCl}, \mathrm{pH}$.

After further washes with deionized water, the cells on glass coverslips were mounted in Entellan (Merck, Milan, Italy) and observed in a Zeiss Axiophot photomicroscope equipped for epifluorescence (Carl Zeiss, Oberkochen, Germany).

\section{FACS analysis}

To detect DNA by FACS, cells were fixed 72 hrs after siRNA transfection; they were washed twice in ice-cold PBS, fixed in ice-cold $70 \%$ ethanol, washed in PBS, resuspended in $2 \mathrm{ml}$ of a solution containing $25 \mu \mathrm{g} / \mathrm{ml}$ of propidium iodide in PBS and $25 \mu \mathrm{l}$ of RNAse $1 \mathrm{mg} / \mathrm{ml}$ in water, and stained overnight at $4^{\circ} \mathrm{C}$ in the dark. Cell cycle analysis was done on at least 10,000 cells for each sample using the FACS Calibur instrument (Becton Dickinson, HO, USA).

To detect cyclin B1 levels by FACS, cells were fixed in $70 \%$ ethanol and stored at $4^{\circ} \mathrm{C}$ for at least one night. They were then washed in cold PBS, permeabilized with $0.25 \%$ Triton X-100 in PBS for 5 min. on ice. Cells were then incubated with $200 \mu \mathrm{l}$ of the anti-cyclin B1 antibody $(0.5 \mu \mathrm{g} / \mathrm{sample}$, clone GNS1 from Pharmingen, San Diego, CA, USA) diluted in rinsing buffer (1\% BSA in PBS), overnight at $4^{\circ} \mathrm{C}$ with gentle agitation. A blank sample was prepared by incubating cells with $200 \mu$ l of isotype lgG instead of cyclin B1. Cells were then washed once before incubation with $200 \mu \mathrm{l}$ of 
Alexa Fluor $488 \mathrm{~F}(\mathrm{ab}) 2$ goat antimouse IgG (Molecular Probes) diluted $1: 500$ in $0.5 \%(v / v)$ Tween-20 in PBS for $1 \mathrm{hr}$, and incubated at room temperature in the dark with gentle agitation. Cells were washed in PBS then stained for $30 \mathrm{~min}$. at room temperature in the dark with a TP3 solution containing $0.5 \mu \mathrm{M}$ TO-PRO-3 in PBS and $25 \mu \mathrm{l}$ RNAse $1 \%$ in PBS. Cell cycle analysis was done with a FACS Calibur instrument (Beckton Dickinson) equipped with a second red iodide laser emitting at $635 \mathrm{~nm}$ and a band pass filter at $661 \pm 16 \mathrm{~nm}$ for TP3 detection.

The active caspase 3 assay was performed as following described. At 72 and $96 \mathrm{hrs}$ after siRNAs transfection, cells were fixed in 1\% paraformaldehyde for $1 \mathrm{hr}$ on ice then in $70 \%$ ethanol, and stored at $4^{\circ} \mathrm{C}$. They were washed once in PBS and permeabilized for $10 \mathrm{~min}$. with $0.004 \%$ saponin in PBS supplemented with $0.5 \%$ BSA and $5 \mathrm{mM}$ EDTA. After centrifugation, the cells were resuspended with $20 \mu \mathrm{l}$ of anti-active caspase-3 PE antibody (Becton Dickinson) and incubated for $1 \mathrm{hr}$ in the dark at room temperature. After washing with PBS, they were analysed with a FACS Calibur instrument (Beckton Dickinson).

\section{Results}

\section{U20S cells lacking Chk1 show proliferation defects and alteration of the cell cycle profile}

To investigate the role of Chk1 in unstressed conditions, we used the siRNA strategy to specifically deplete this protein kinase in U20S cells. Chk1 protein levels were efficiently down-regulated from $24 \mathrm{hrs}$, and the effect persisted up to $144 \mathrm{hrs}$ after transfection, compared to the scramble siRNA transfected cells (Fig. 1A and data not shown).

Chk1 depletion in U20S cells has been recently reported [13] and we could confirm an increase in H2AX phosphorylation starting 48 hrs after siRNA Chk1 transfection, as compared with the scramble siRNA transfected cells, and persisting up to $72 \mathrm{hrs}$ (Fig. 1B).

We investigated whether Chk1 depletion affected U2OS cell proliferation. Transfection with the Chk1 siRNA strongly inhibited cell proliferation, compared to the scramble transfected cells, starting from 72 hrs and more clearly at 96 hrs (Fig. 1C). Cell cycle analysis showed an increase in the amount of cells in S and G2/M phases 72 hrs after transfection (Fig. 1D).

The effects of Chk1 depletion in U2OS cells are different if compared with previous data $[10,11]$ and with the data showed in Fig. 2, reporting that Chk1 depletion did not alter the cell proliferation and the cell cycle profile in various human cancer cell lines (Fig. 2).

\section{Chk1 depletion in U20S cells leads to accumulation of cells with 4N DNA content but not of mitotic cells}

To better define the nature of the accumulation of U20S cells lacking Chk1 in S and G2/M phases of the cell cycle, we analysed the cyclin B1 levels by FACS. Cyclin B1 is specifically expressed in late $S$ phase and is degraded by E3 ubiquitin ligase activity before anaphase [4]. Efficient depletion of Chk1 72 hrs after siRNA transfection was confirmed by Western blot analysis (Fig. 3A). The Chk1depleted cells at this time showed a consistent accumulation of cells with $4 \mathrm{~N}$ DNA content, suggesting they were at the G2/M phase of the cell cycle $(42.7 \%$ versus $17.9 \%$ in scramble transfected cells). However, the number of cells positive for cyclin B1 and 4N DNA content (R2, R3 and R4 boxes, Fig. 3B) was comparable in the scramble and Chk1 siRNA samples. These findings, together with the considerable amount of cyclin B1 negative cells in G2/M in the siRNA Chk1 transfected sample (R1 box, Fig. 3B), suggested that part of the apparent G2/M population might be tetraploid in G1. This was confirmed by immunofluorescence (IF) staining using the antibody against phospho- $\mathrm{H} 3$, a specific mitotic marker, which showed no accumulation of cells in mitosis and no apparent abnormal mitotic phenotype in Chk1-depleted U2OS cells (Fig. 3C). There were less than $3 \%$ of phospho-H3 positive cells in the scramble and in the Chk1-depleted cells thus confirming that the accumulation of cells with 4N DNA content was not linked to a block in mitosis.

\section{Chk1-depleted U20S cells show an accumulation of abnormal micro-polynucleated cells that leads to cell death by a predominantly apoptosis independent mechanism}

To investigate whether the lack of proliferation of Chk1-depleted cells was due to apoptosis, sulforodamine/DAPI staining was done. We observed both clearly apoptotic (thin arrow, Fig. 4A) and abnormal micropolynucleated morphologies (thick arrow, Fig. 4A) in siRNA Chk1 transfected cells, while the siRNA scramble transfected cells looked morphologically normal (Figs $3 \mathrm{C}$ and 4A). Staining with lamin B showed apoptotic cells, which had lost their nuclear envelope integrity (Fig. 4B, thin arrows), while the micro-polynucleated cells still had intact lamin B (Fig. 4B, thick arrow). To quantify the rate of apoptosis, we carried out flow cytometric analysis on cells stained with an antibody that detected cleaved caspase-3 (Fig. 4C). At 72 and 96 hrs after Chk1 depletion, there were $6 \%$ and $20 \%$ of dead cells compared to the scramble siRNA transfected cells (Fig. 4C). In fact the percentage of viable cells were, respectively, of $96.10 \%$ (scramble) and $90.43 \%$ (Chk1) at $72 \mathrm{hrs}$ and $95.55 \%$ (scramble) and $76.38 \%$ (Chk1) at $96 \mathrm{hrs}$ after siRNA transfection (gate R1, Fig. 4C). This suggested that cell death and/or proliferation defects, inhibiting the growth of siRNA Chk1 transfected cells, were not uniquely associated with apoptosis. The micro-polynucleated cells were larger (4A, upper panel). Interestingly, a subpopulation of the polynucleated cells were in the last step of cytokinesis with the two daughter cells containing micronuclei with decondensed DNA but still not completely separated into two separate cells (Fig. 5A, lower panel). In addition, these same cells showed an Aurora B positive signal in the region corresponding to the chromosome bridge (Fig. 5B). The morphologies observed in siRNA Chk1-treated U20S cells, shown in Figs 4 and 5 (panels $A$ and $B$ ), might represent cells with a $4 \mathrm{~N}$ DNA content exiting from mitosis but not dividing correctly, 
A

scramble siRNA

Chk1 siRNA

time (h)

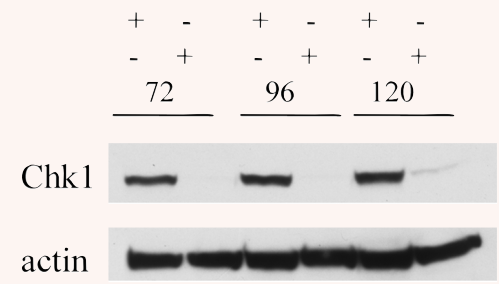

B

scramble siRNA

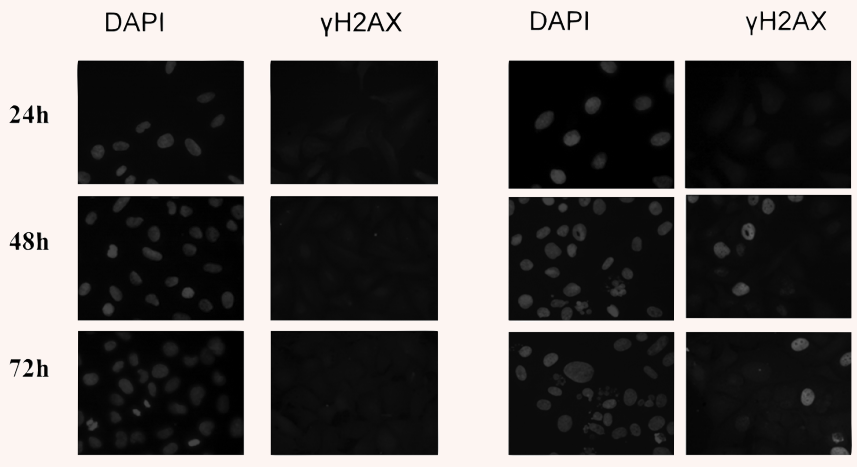

C

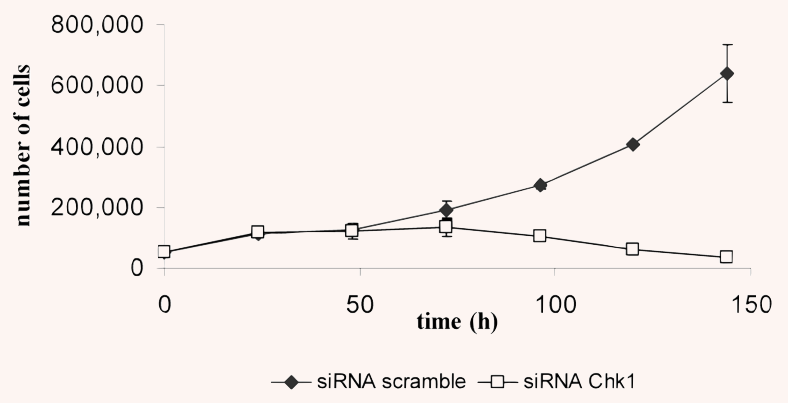

D

siRNA scramble siRNA Chk1

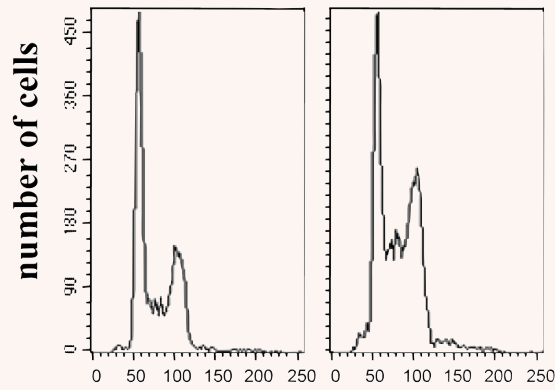

DNA Content
Fig. 1 Growth survival and cell cycle profile of U2OS Chk1-depleted cells. (A) Western blot analysis of Chk1 and actin in U2OS cells from 72 to $120 \mathrm{hrs}$ afer siRNAs transfection. (B) $\mathrm{pH} 2 \mathrm{AX}$ immunofluorescence staining in scramble and Chk1 siRNA-transfected cells 24,48 and 72 hrs after transfection. DAPI staining is also shown. (C) Cell growth curve. The data represent the mean \pm S.D. of two independent experiments. (D) DNA content by FACS analysis. 
A

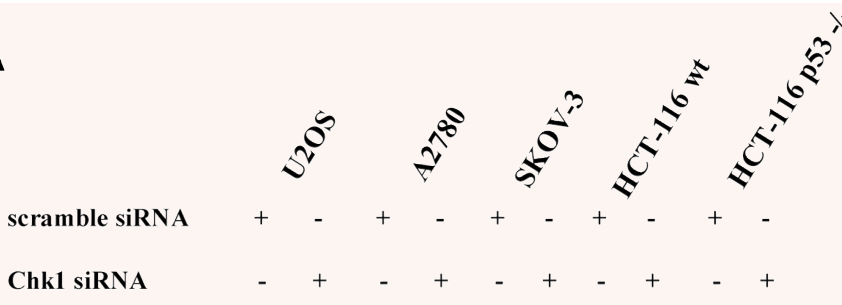

Chk1 -

Ran

B

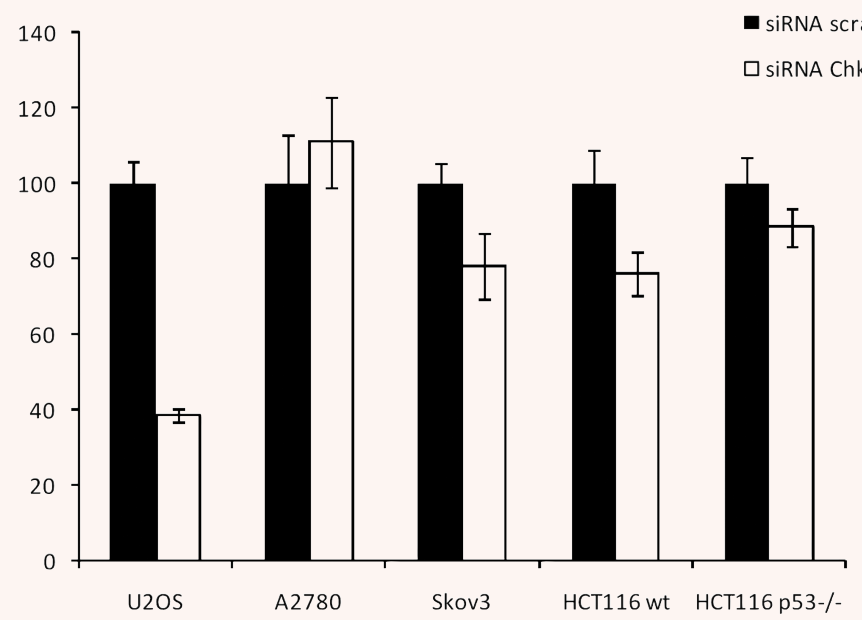

C

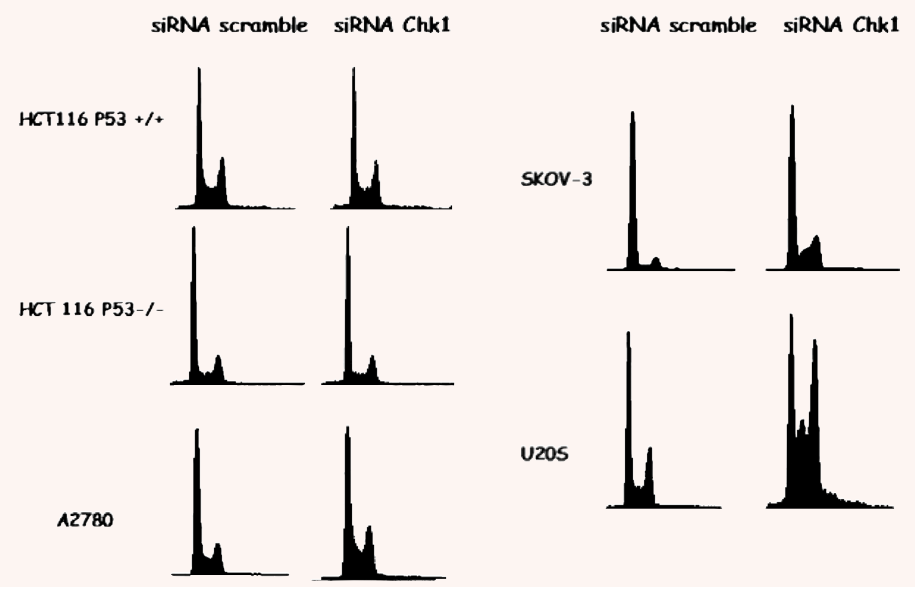

Fig. 2 Effects of Chk1 depletion in various human cancer cell lines. (A) Western blot analysis of Chk1 and Ran in U20S, A2780, SKOV-3, HCT-116 wt and HCT-116 p53 -/- 96 hrs after siRNA transfection. (B) Schematic representation of the percentage of cells surviving at 96 hrs after transfection with siRNA Chk1. Data are expressed as relative percentage of scramble transfected cells and are the mean \pm S.D. of two independent experiments. (C) FACS analysis performed on the different human somatic cancer cell lines at $72 \mathrm{hrs}$ after transfection with either scramble or Chk1 siRNA. 
A

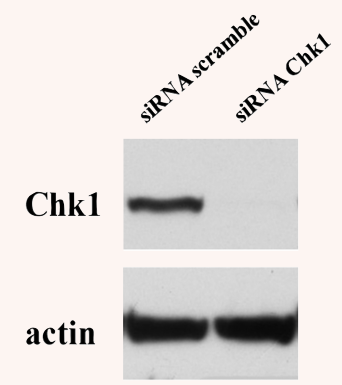

B

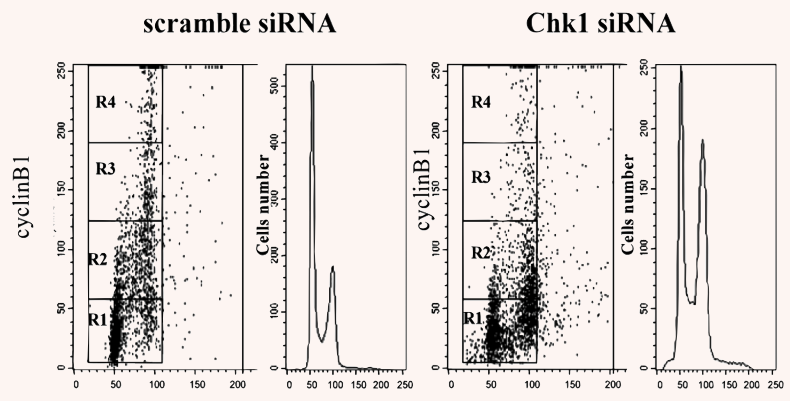

DNA Content

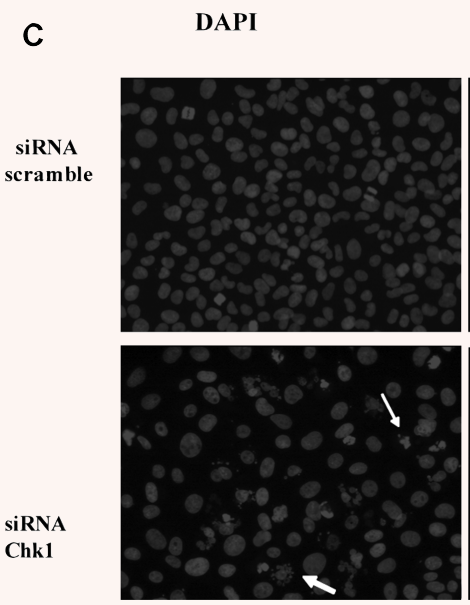

phosphorylatedserine 10 Histone 3
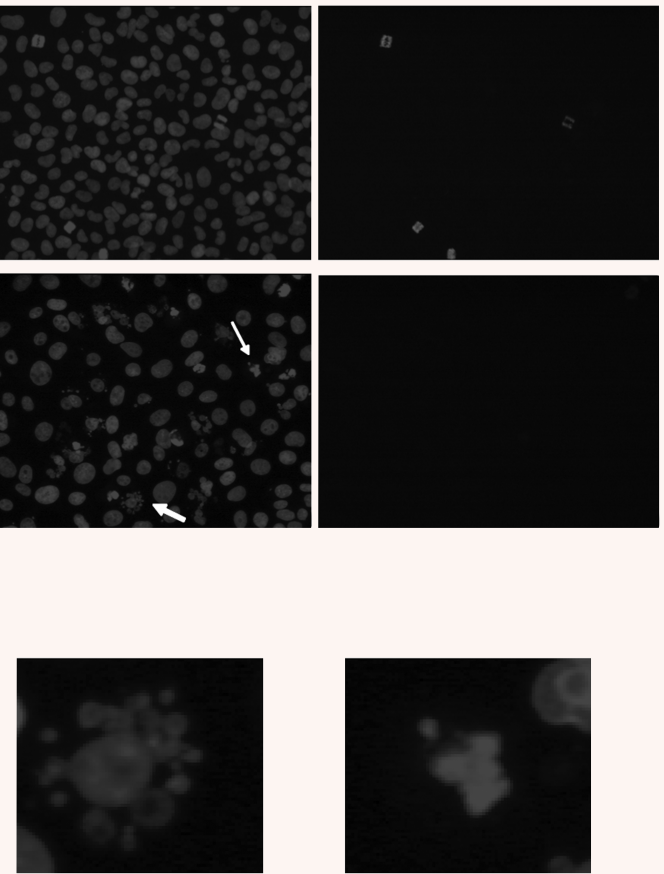

Polymicronucleated morphology

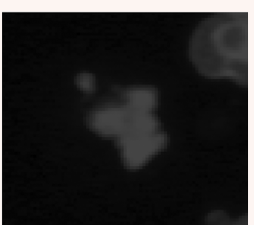

Apoptotic morphology
Fig. 3 Accumulation of U20S cells with a 4N DNA content. (A) Western blot analysis of Chk1 and actin in U2OS cells 72 hrs after siRNA transfection. (B) Cell cycle specific expression of cyclin B1 72 hrs after siRNA transfection. Cells were double stained with cyclinB1 and ToPro and FACS analysis was done at $72 \mathrm{hrs}$ after transfection with siRNAs. The bi-parametric analysis and the simple DNA content are presented for each sample. The R1 box represents the cyclin B1 negative population and R2, R3 and R4 boxes show the different levels of cyclin B1 expression. (C) Phosphorylated serine 10 Histone 3 and DAPI immunofluorescence in U20S cells 72 hrs after siRNA transfection. Arrows point to siRNA Chk1 transfected cells with abnormal morphology, which are more clearly represented in the lower part of the figure. Thick arrow: polymicronucleated morphology; thin arrow: apoptotic morphology. 
A
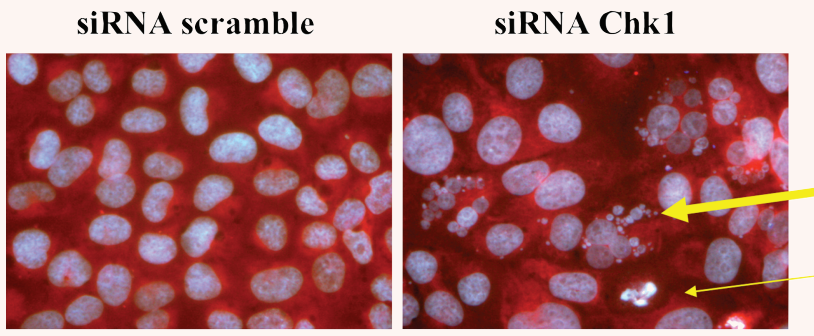

B
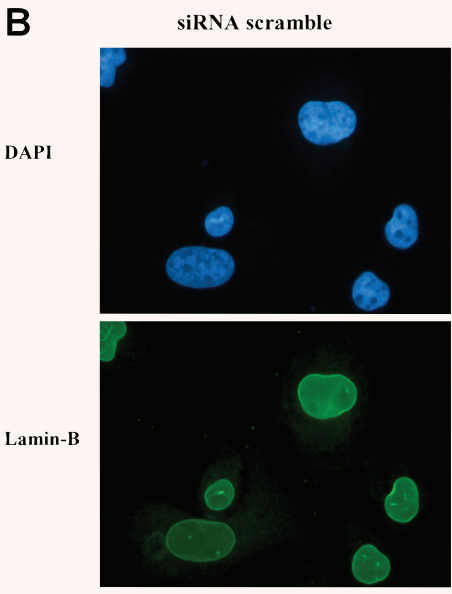

siRNA Chk1

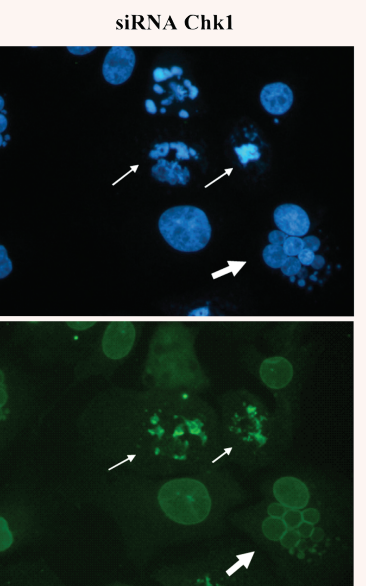

C
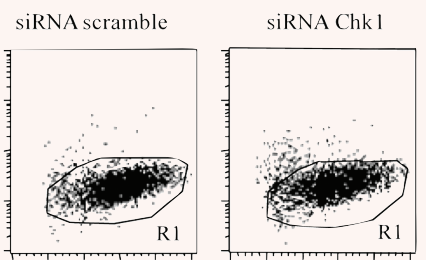

$72 \mathrm{~h}$
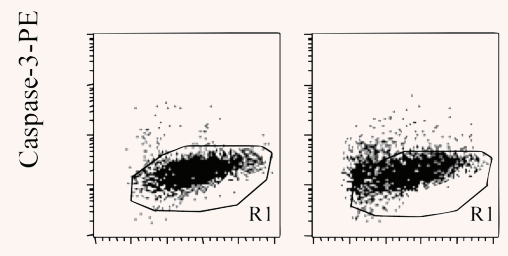

$96 \mathrm{~h}$

Fig. 4 Chk1-depleted cells die by a mechanism predominantly apoptosis independent (A) Sulforodamine-DAPI staining of U20S cells 72 hrs after siRNA transfection. The thin yellow arrow indicates a typical apoptotic morphology and the thick one points to a polynucleated cell. (B) Lamin B and DAPI staining in U20S cells 72 hrs after transfection. The thin white arrows show the breaking down nuclear envelopes in apoptotic cells and the thicker one points to the intact nuclear envelope of a micronucleated cell. (C) Bi-parametric forward scatter/caspase-3-PE analysis in U20S cells evaluated at 72 and $96 \mathrm{hrs}$ after transfection. The amount of viable cells is represented by the gate R1. U20S cells treated with staurosporine (A) were included in the experiment as positive control (percentage of viable cells in positive control sample: $24.17 \%$ ).

leading to accumulation of tetraploid cells in G1. Concurrent with this, we also observed high levels of p53 and p21 and phosphorylation in ser 15 residue of $p 53$ in the siRNA Chk1 transfected cells 72 hrs after transfection (at which time there was clear accumulation of 4N DNA content), suggesting that the G1 tetraploid block may occur through p53-dependent activation of p21 (Fig. 5C). Transfection of U2OS cells with a second siRNA directed against a different Chk1 siRNA target confirmed the results just described (data not shown) thus suggesting that the effect observed is specific and uniquely attributable to the lack of Chk1.

\section{Chk1-depleted U20S cells are unable to arrest in mitosis following spindle disruption by nocodazole}

To investigate whether the abnormal phenotype in Chk1-depleted U20S cells was the consequence of a defect in the spindle checkpoint, U20S cells transfected with either scramble or siRNA Chk1 were treated with $75 \mathrm{nM}$ of Noc at 48 hrs after transfection for $20 \mathrm{hrs}$, then washed and incubated in Noc-free medium for another 7 hrs. As shown in Fig. 6A, after 20 hrs of Noc treatment, most of the siRNA scramble transfected cells had rounded up having the typical mitotic morphology, which it was not observed in the siRNA Chk1 transfected cells. To quantify the cells in mitosis, cells treated or not with Noc were stained with phospho- $\mathrm{H} 3$ and analysed by IF. As shown in Fig. 6B, less than $2.5 \%$ of asynchronous untreated cells transfected with either scramble or Chk1 siRNA appeared phospho-H3 positive. However, 20 hrs of Noc induced an obvious accumulation of cells in mitosis in siRNA scramble transfected cells (approximately 40\%) whereas the proportion of mitotic cells in Chk1-depleted cells treated with Noc was much lower (approximately 10\%). At 7 hrs after Noc release, the U20S siRNA scramble transfected cells had completely re-entered the cell cycle, exiting from the mitotic block. At this time-point, 
A

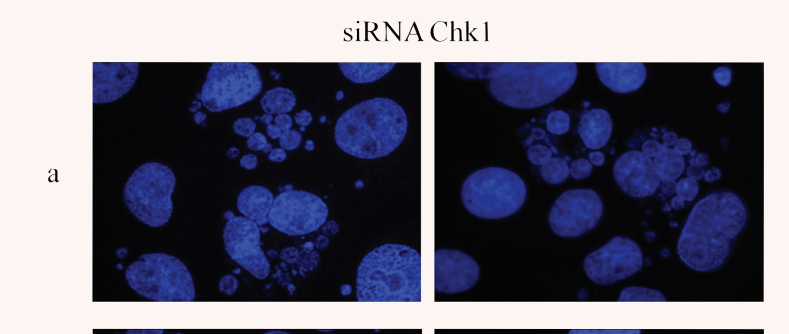

$\mathrm{b}$

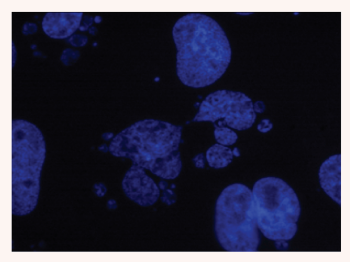

B
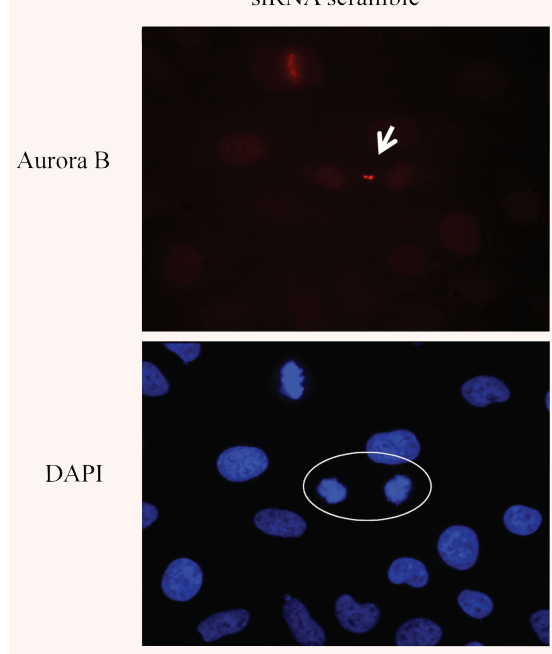

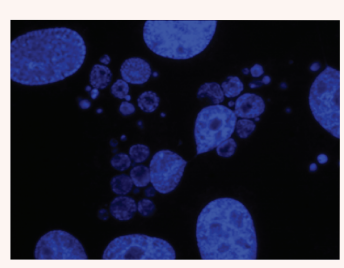

siRNA Chk 1
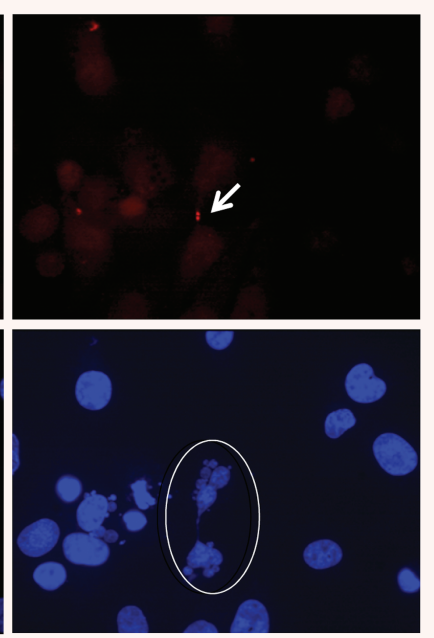

C

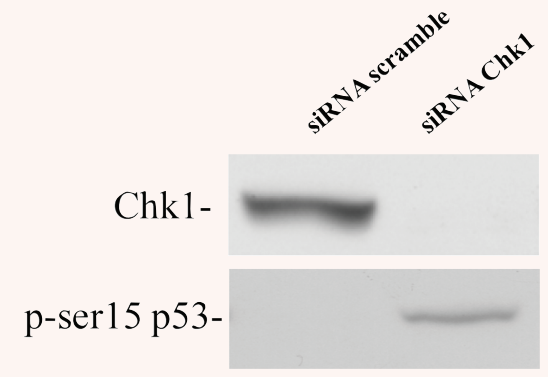

p53-

p21 -

actin -
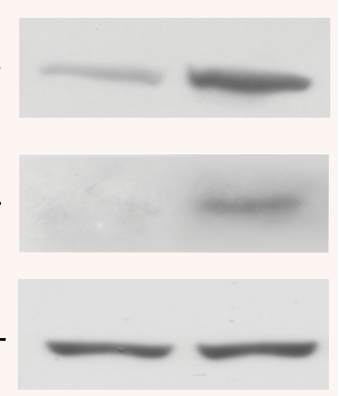

Fig. 5 Phenotypical characterization of siRNA Chk1 transfected U20S cells. (A) DAPI staining of U20S cells 72 hrs after transfection with siRNA Chk1. The upper panels (a) give two examples of the massive presence of the micropolynucleated phenotype and the lower panels (b) show micropolynucleated cells appearing at the last stage of cytokinesis with nuclei still attached. (B) Aurora B and DAPI staining of U2OS cells 72 hrs after siRNAs transfection. The white arrows indicate the Aurora B signal in the corresponding chromosome bridge of dividing cells (white circle). (C) Western blot analysis of Chk1, p-ser15 p53, p53, p21 and actin protein levels of U20S cells 72 hrs after siRNAs transfection.

fewer than $2 \%$ of siRNA Chk1 transfected cells were in mitosis both with and without Noc treatment. Figure $6 \mathrm{C}$ shows the cell cycle profile of U20S cells transfected with either scramble or siRNA Chk1 at different times after Noc treatment: 48 hrs after transfection, when Noc treatment started the cell cycle profiles were very similar in both cell groups. After $20 \mathrm{hrs}$ of incubation in Noc $(0 \mathrm{hr})$, the cells transfected with the scramble siRNA had a large proportion with a $4 \mathrm{~N}$ DNA content indicative of mitotic arrest. In contrast, after 20 hrs in Noc the cells transfected with the Chk1 siRNA had a large percentage with less than 4N DNA content (G1 and $S$ phase) as well as an increase in the population with a 4 N DNA content. By 7 hrs after removal of Noc, the scram- ble siRNA transfected cells had exited mitotis and had re-entered the cell cycle, while the siRNA Chk1 transfected cells showed a persistent peak with a 4N DNA content, similar to the cells that had received Chk1 siRNA but not Noc. The lack of accumulation of phospho-H3 positive Chk1 siRNA transfected cells (Fig. 6B), together with the cell cycle analysis (shown in Fig. 6C), strongly suggests that the Chk1-depleted cells with the G2/M DNA content is not related to a Noc-induced mitotic arrest.

To corroborate this finding, we analysed the expression of cyclin B1, a mitotic cyclin and cyclin E, a G1 cyclin, by Western blot (Fig. 6D). As expected, cyclin B1 accumulated in scramble siRNA transfected cells 20 hrs after Noc treatment (Figs. 6D 

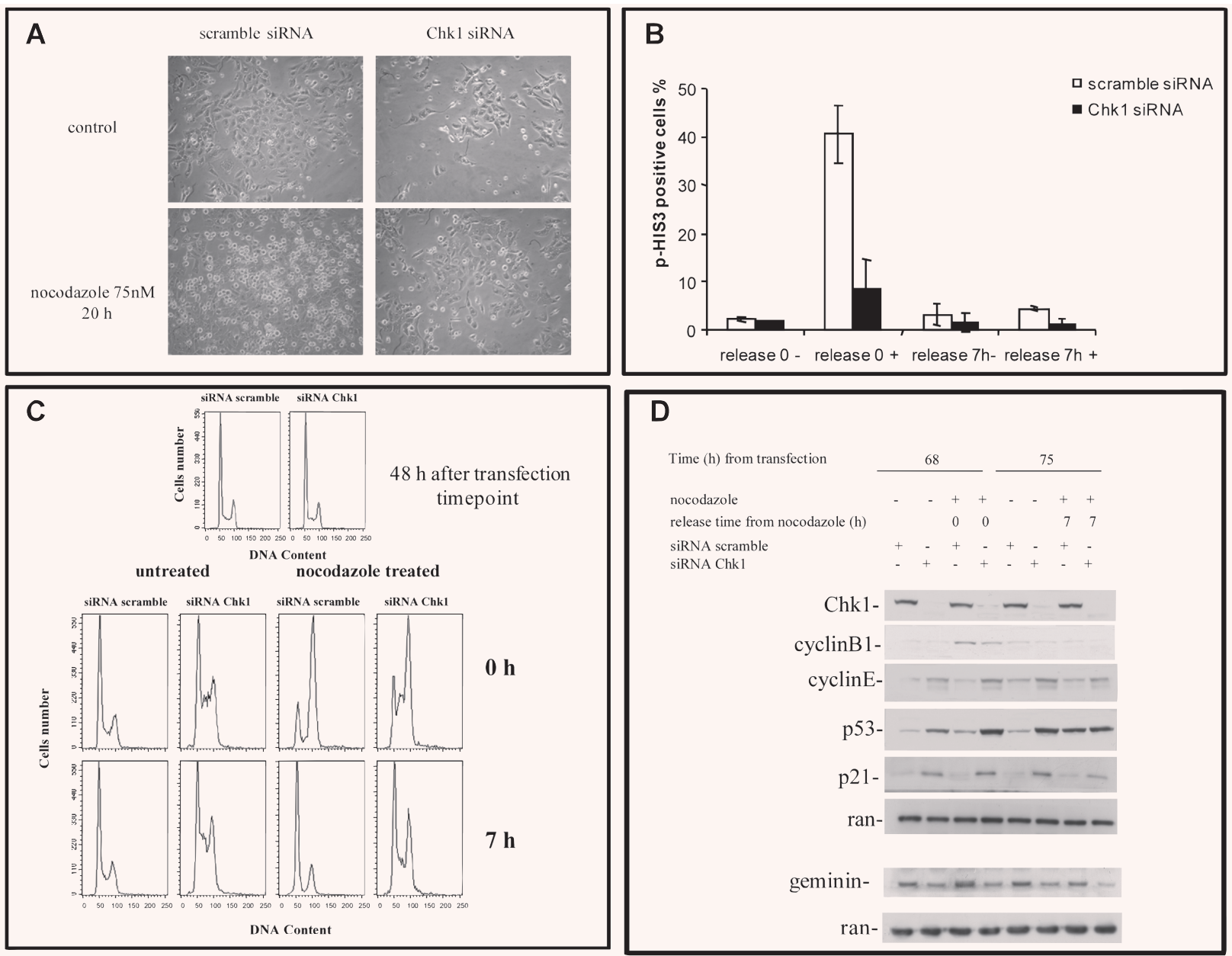

Fig. 6 Effects of nocodazole in siRNA scramble or siRNA Chk1 transfected U2OS cells. (A) DIC microscopy pictures of control and Noc treated (20 hrs $75 \mathrm{nM}$ ), scramble and Chk1 siRNA transfected U20S cells. (B) Mitotic index analysis of cells either treated (+) or not (-) with Noc (released 0 and $7 \mathrm{hrs}$ after $20 \mathrm{hrs}$ of treatment $75 \mathrm{nM}$ ). Cells were fixed with paraformaldehyde and stained with the phospho-H3 antibody and DAPI. The data are the mean \pm S.D. of three independent experiments. The percentage of phospho-H3 positive cells was calculated on at least 300 cells. (C) FACS analysis showing the DNA profile at the start of Noc treatment (48 hrs after transfection), at the end, and $7 \mathrm{hrs}$ after Noc release. (D) Western blot analysis of Chk1, cyclinB1, cyclinE, p53, p21, geminin and Ran protein levels at the end and 7 hrs after 20 hrs of Noc treatment. Geminin and its relative Ran were detected on a different SDS-PAGE (lower panel).

and 7A). No accumulation of cyclin B1 was detected in any other scramble or Chk1 siRNA transfected samples treated or not with Noc (Fig. 6D), suggesting again that the higher 4N DNA content in siRNA Chk1 transfected cells is not related to an accumulation of mitotic cells. Cyclin E levels, however, appeared higher in all the siRNA Chk1 transfected samples than the siRNA scramble transfected cells. To further corroborate the fact that Chk1-depleted U20S cells were in G1 phase, geminin levels were investigated (Fig. 6D, lower part). Geminin fluctuates throughout the cell cycle with its levels lowest at G1 and consistently elevated in S and G2. It is degraded by the anaphase-promoting complex (APC) in mitosis [15]. No accumulation of geminin could be detected in any siRNA Chk1 transfected samples treated or not with Noc as compared to siRNA scramble transfected cells. Thus Chk1depleted cells, despite their DNA content in the G2 or M phase of the cell cycle, express high levels of cyclin E, a G1 marker and low levels of geminin, an S and G2 marker. Once again, p53 and p21 protein levels were increased in Chk1-depleted cells, treated or not with Noc, suggesting that the G1 block in the tetraploid status may occur through the p53 pathway. The high p53 levels after Noc in the siRNA scramble transfected cells has already been documented [16]. Our data suggest that Chk1 depletion causes U20S to fail to activate the spindle checkpoint induced by unattached kinetochores. 
A

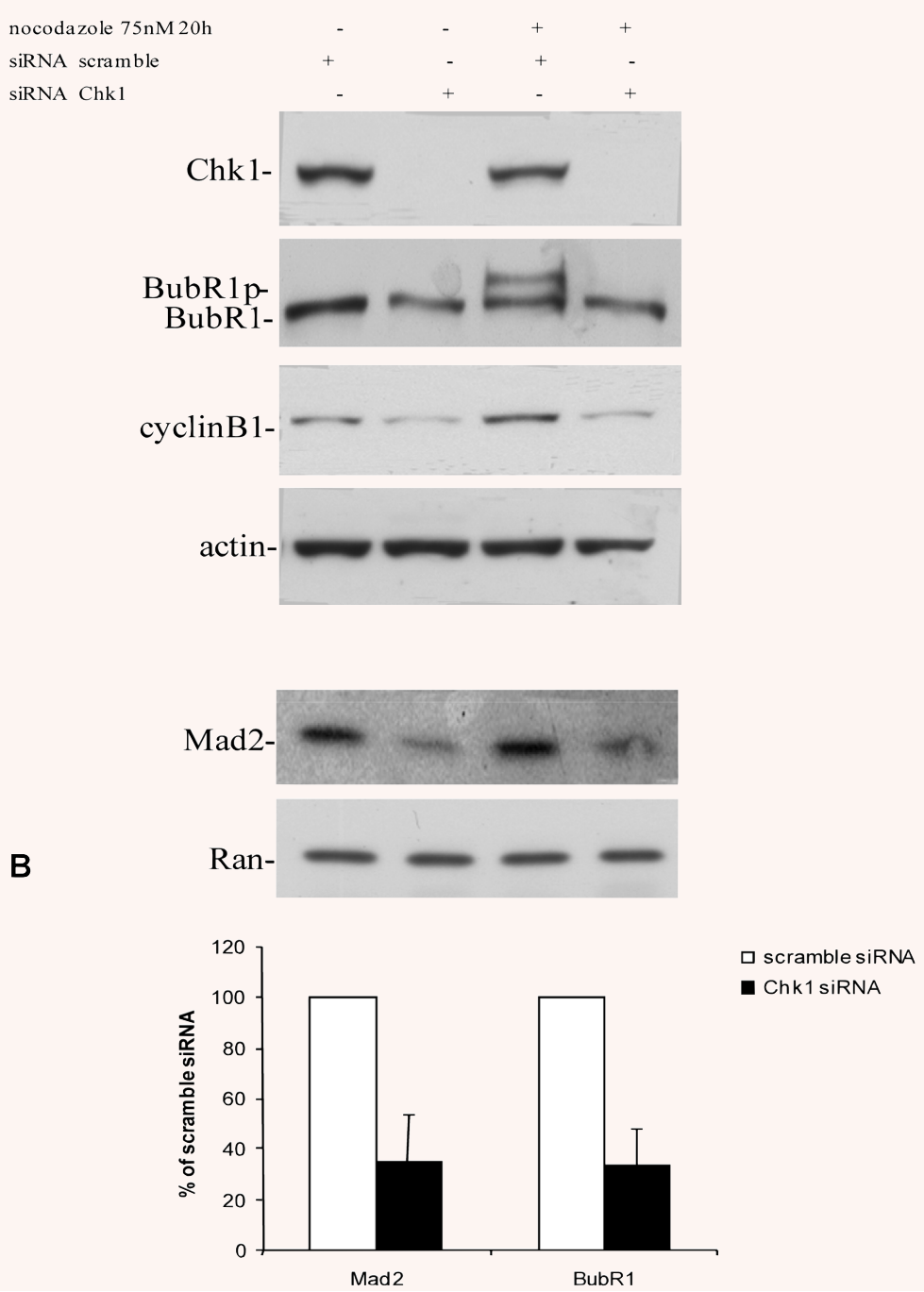

Fig. 7 Mitotic spindle checkpoint protein levels in Chk1-depleted U2OS cells. (A) Western blot analysis of Chk1, BubR1, cyclinB1, actin, Mad2 and Ran protein levels in siRNA scramble and Chk1 transfected cells, either treated or not with Noc $75 \mathrm{nM}$ for 20 hrs. Mad2 and Ran protein levels were detected on $10 \%$ SDS-PAGE and the other proteins on $6 \%$ SDSPAGE. (B) Densitometric analysis of Mad2 and BubR1 protein levels in U20S cells 72 hrs after siRNA Chk1 transfection, compared with the siRNA scramble transfected cells. Data are expressed as percentage of siRNA scramble relative controls, representing the ratio between Mad2 or BubR1 and Ran or actin, respectively. Error bars show the standard deviation from the mean of three independent experiments.

\section{Possible requirement of Chk1 in U20S cells to activate the mitotic spindle checkpoint proteins Mad2 and BubR1}

To investigate the molecular mechanisms of the accumulation of morphologically abnormal G1 tetraploid cells after Chk1 depletion, and the lack of mitotic arrest in these cells after Noc treatment, we examined the protein levels of two of the principal components of the mitotic spindle checkpoint Mad2 and BubR1 [17]. Mad2 and BubR1 protein levels were lower in the Chk1-depleted cells $72 \mathrm{hrs}$ after siRNA Chk1 transfection than in siRNA scramble transfected cells. Chk1 depletion also prevented the accumulation of these proteins following Noc treatment. BubR1 phosphorylation after Noc was also absent in the Chk1-depleted cells (Fig. 7A). Figure 7B shows densitometric analysis of three independent experiments
72 hrs after siRNA scramble or Chk1 transfection. Mad2 and BubR1 protein levels decreased by $75 \%$ and $77 \%$ in siRNA Chk1 transfected cells compared to siRNA scramble transfected ones. Real-time PCR analysis detected no significant decrease in Mad2 and BubR1 mRNAs (data not shown).

\section{Discussion}

While the role of Chk1 as a key regulator of the DNA damage and replication checkpoint has been established, evidence of its role in unstressed conditions is more controversial. We used the selective siRNA technique to completely deplete the expression of Chk1 in the osteosarcoma cell line U2OS. Increased $\gamma \mathrm{H} 2 \mathrm{AX}$ phosphorylation 
was observed, as previously reported for this cell line [13]. However, unlike previous studies using Chk1 down-regulation, here we noted that, starting from 72 hrs after Chk1 down-regulation, cells stopped growing and died both by apoptosis and by an apoptosis independent mechanism, as a consequence of accumulation of abnormal giant, polynucleated tetraploid cells, with evident G2/M accumulation observed by cell cycle analysis. These observations contrast with previous data and with data showed in Fig. 2, reporting that siRNA Chk1 depletion did not alter cell proliferation or the cell cycle profile in other human cancer cell lines with a different status of p53 under unstressed conditions $[10,11]$, suggesting that the consequences of Chk1 depletion may differ depending on the experimental system studied.

The results in Chk1-depleted U20S cells might partly be a direct consequence of entering mitosis with damaged DNA. Chk1depleted U20S cells have a high incidence of DNA strand breaks resulting from increased initiation of DNA replication [13]. The lack of Chk1 would not lead to an efficient S or G2 checkpoint and efficient repair of DNA damage in these cell cycle phases. Cells that enter mitosis with extensive DNA damage could encounter problems with segregation of abnormal chromosomes, which could lead to defects in mitotic exit and cytokinesis with consequent accumulation of $\mathrm{G} 1$ tetraploid cells. These giant micropolynucleated cells are prevented from cycling again in a p53- and p21-dependent manner and subsequently die because of the accumulation of severe DNA damage. The p53- and p21-dependent activation of the $\mathrm{G} 1$ checkpoint after Chk1 depletion might result in tetraploid cells and may be how Chk1-depleted cells attempt to repair the damage accumulated in previous cell cycle stages. The lack of a mitotic block in the presence of abnormal DNA structures in Chk1-depleted U20S cells suggests that, as has already been described in fission and budding yeast $[18,19]$, the Chk1 protein kinase has a major role not only in the $S$ and $G 2$ checkpoints but also in sustaining activation of the spindle checkpoint whenever cells with damaged DNA enter mitosis. These data thus offer a new example of conservation of the Chk1 pathway in eukaryotic systems and new evidence of a role of Chk1 in the mitotic spindle checkpoint in human cells.

The results described are in contrast with recent observations in HeLa cells [5]. This might be explained by the different experimental systems and further strengthens the evidence that the lack of Chk1 may have different consequences depending on the genetic background of the experimental system.

We found that down-regulation of Chk1 in U20S cells led to loss of viability, accumulation of tetraploid cells after mitotic failure and inability to activate the spindle checkpoint and arrest in mitosis after treatment with the microtubule destabilizing drug Noc. The mitotic spindle checkpoint acts by inhibiting the APC required to promote degradation of cyclin $B 1$ and cohesin $[4,20]$. The APC is inhibited by preventing its association with Cdc20 [17, 20]. This requires both the checkpoint proteins Mad2 and BubR1, which inhibit the APC whenever a misaligned or unattached chromosome is detected, thus arresting cells at the metaphaseanaphase transition $[4,17]$. We found that Chk1 down-regulation in U20S cells led to lower levels of both Mad2 and BubR1. It has been previously reported that the lack of Mad2 in human cells led to a failure of metaphase block after Noc treatment and to an accumulation of micropolynucleated cells with aneuploid DNA content [21]. Mad2, like Chk1, is essential for mouse embryogenesis as Mad2 -/- KO mice die at E6.5 as a consequence of chromosome mis-segregation and apoptosis [22]. This matches with the results after Chk1 depletion and, along with the finding of Chk1-dependent down-regulation of Mad2 protein, supports the idea that Chk1 and Mad2 may be involved in the same pathway regulating the accurate progression of mitosis. Experimental evidence supports this relationship in fission yeast where the Chk1 dependent delay of metaphase-anaphase transition in mitotic cells with damaged DNA was Mad2 dependent [18]. It will be important to clearly define if a direct or indirect interaction between Chk1 and Mad2 is occurring. Analysis of the Mad2 protein sequence revealed a putative Chk1 phosphorylatable site at ser195 position [23]. Studies are now in progress to see whether Chk1 has a part in this Mad2 post-translational modification. The few data available on how Mad2 phosphorylation functions during the cell cycle are still incomplete, but ser195 is one putative phosphorylation site [24].

BubR1, together with Mad2, is mainly involved in the mitotic spindle checkpoint. Both proteins are recruited to unattached kinetochores, but only BubR1 appears to be recruited to kinetochores in the absence of tension [25]. Chk1 is required for phosphorylation and localization of BubR1 to kinetochores in order to sustain mitotic delay after activation of the mitotic spindle checkpoint by taxol, a drug that stabilizes microtubules and increases aberrant kinetochore microtubule attachments, but not by Noc [6]. Here we found low BubR1 protein levels in Chk1-depleted U20S cells, suggesting that its stability too may be directly regulated by Chk1. Nonetheless, in our experimental system a Chk1-dependent BubR1 phosphorylation was also observed after Noc treatment. Furthermore, differently to what has been reported by others [6], Chk1-depleted U20S cells were unable to arrest in mitosis after this treatment, which prevents microtubule polymerization and spindle formation delaying the metaphase-anaphase transition, with persistent high levels of cyclin B1. Chk1-depleted U20S cells did not accumulate cyclin B1 even in the presence of Noc (Figs 6D and 7A). Low levels of geminin in Chk1-depleted U20S cells corroborated the fact that these cells were not accumulated before mitosis in $S$ and $G 2$ phases. In addition, the high expression of p21 and cyclin E after Chk1 depletion further suggests that these cells have by-passed the metaphase block aberrantly and are blocked in G1 phase, giving rise to a tetraploid population. The mechanism by which Chk1 inhibition leads to low levels of Mad2 and BubR1 has still to be elucidated. However, our findings clearly show that Chk1 is required not only in $\mathrm{S}$ and $\mathrm{G} 2$ checkpoints but also for activation of the mitotic spindle checkpoint.

These results support an important role for Chk1 in unstressed conditions and merit more detailed investigation since Chk1 has been proposed as a potential drug target for anticancer therapies. As the death of non-tumour proliferating cells limits the efficacy of most current chemotherapies, if Chk1 inhibition 'per se' 
causes toxicity, this may limit its effectiveness. However, the fact that U20S cells are so sensitive to Chk1 depletion suggests that some tumours at least might be very responsive to Chk1 inhibitors.

\section{Acknowledgements}

The generous contribution of the Italian Association for Cancer Research and of FIRBMIUR is gratefully acknowledged.

\section{References}

1. Walworth N, Davey S, Beach D. Fission yeast chk1 protein kinase links the rad checkpoint pathway to cdc2. Nature. 1993; 363: 368-71.

2. Takai H, Tominaga $\mathrm{K}$, Motoyama $\mathrm{N}$, et al. Aberrant cell cycle checkpoint function and early embryonic death in Chk1(-/-) mice. Genes Dev. 2000; 14: 1439-47.

3. Zachos G, Rainey MD, Gillespie DA. Chk1-dependent S-M checkpoint delay in vertebrate cells is linked to maintenance of viable replication structures. Mol Cell Biol. 2005; 25: 563-74.

4. Bharadwaj R, Yu H. The spindle checkpoint, aneuploidy, and cancer. Oncogene. 2004; 23: 2016-27.

5. Tang J, Erikson RL, Liu X. Checkpoint kinase 1 (Chk1) is required for mitotic progression through negative regulation of polo-like kinase 1 (Plk1). Proc Natl Acad Sci USA. 2006; 103: 11964-9.

6. Zachos G, Black EJ, Walker M, et al. Chk1 is required for spindle checkpoint function. Dev Cell. 2007; 12: 247-60.

7. Liu Q, Guntuku S, Cui XS, et al. Chk1 is an essential kinase that is regulated by Atr and required for the $\mathrm{G}(2) / \mathrm{M}$ DNA damage checkpoint. Genes Dev. 2000; 14: 1448-59.

8. Niida H, Tsuge S, Katsuno $\mathrm{Y}$, et al. Depletion of Chk1 leads to premature activation of Cdc2-cyclin B and mitotic catastrophe. J Biol Chem. 2005; 280: 39246-52.

9. Zachos G, Rainey MD, Gillespie DA. Chk1-deficient tumour cells are viable but exhibit multiple checkpoint and survival defects. EMBO J. 2003; 22: 713-23.
10. Carrassa L, Broggini M, Erba E, Damia G. Chk1, but not Chk2, is involved in the cellular response to DNA damaging agents: differential activity in cells expressing or not p53. Cell Cycle. 2004; 3: 1177-81.

11. Chen Z, Xiao Z, Chen J, et al. Human Chk1 expression is dispensable for somatic cell death and critical for sustaining G2 DNA damage checkpoint. Mol Cancer Ther. 2003; 2: 543-8.

12. Lam MH, Liu Q, Elledge SJ, Rosen JM. Chk1 is haploinsufficient for multiple functions critical to tumor suppression. Cancer Cell. 2004; 6: 45-59.

13. Syljuasen RG, Sorensen CS, Hansen LT, et al. Inhibition of human Chk1 causes increased initiation of DNA replication, phosphorylation of ATR targets, and DNA breakage. Mol Cell Biol. 2005; 25: 3553-62.

14. Schwarz DS, Hutvagner G, Du T, et al. Asymmetry in the assembly of the RNAi enzyme complex. Cell. 2003; 115: 199-208.

15. Tachibana KE, Gonzalez MA, Coleman N. Cell-cycle-dependent regulation of DNA replication and its relevance to cancer pathology. J Pathol. 2005; 205: 123-9.

16. Ciciarello M, Mangiacasale R, Casenghi $\mathbf{M}$, et al. p53 displacement from centrosomes and p53-mediated G1 arrest following transient inhibition of the mitotic spindle. J Biol Chem. 2001; 276: 19205-13.

17. Davenport J, Harris LD, Goorha R. Spindle checkpoint function requires Mad2-dependent Cdc20 binding to the Mad3 homology domain of BubR1. Exp Cell Res. 2006; 312: 1831-42.
18. Collura A, Blaisonneau J, Baldacci G, Francesconi $\mathbf{S}$. The fission yeast Crb2/Chk1 pathway coordinates the DNA damage and spindle checkpoint in response to replication stress induced by topoisomerase I inhibitor. Mol Cell Biol. 2005; 25: 7889-99.

19. Searle JS, Schollaert KL, Wilkins BJ, Sanchez Y. The DNA damage checkpoint and PKA pathways converge on APC substrates and $\mathrm{Cdc} 20$ to regulate mitotic progression. Nat Cell Biol. 2004; 6: 138-45.

20. Fang G, Yu H, Kirschner MW. Control of mitotic transitions by the anaphase-promoting complex. Philos Trans $R$ Soc Lond B Biol Sci. 1999; 354: 1583-90.

21. Michel LS, Liberal V, Chatterjee A, et al. MAD2 haplo-insufficiency causes premature anaphase and chromosome instability in mammalian cells. Nature. 2001; 409: 355-9.

22. Dobles M, Liberal V, Scott ML, Benezra R, Sorger, PK. Chromosome missegregation and apoptosis in mice lacking the mitotic checkpoint protein Mad2. Cell. 2000; 101: 635-45.

23. Hutchins JR, Hughes M, Clarke PR. Substrate specificity determinants of the checkpoint protein kinase Chk1. FEBS Lett. 2000; 466: 91-5.

24. Wassmann K, Liberal V, Benezra R. Mad2 phosphorylation regulates its association with Mad1 and the APC/C. EMBO J. 2003; 22: 797-806.

25. Fang G. Checkpoint protein BubR1 acts synergistically with Mad2 to inhibit anaphase-promoting complex. $\mathrm{Mol} \mathrm{Biol}$ Cell 2002; 13: 755-66. 